\title{
Embarazo cornual: tratamiento médico con dosis única de metotrexato
}

\author{
Paolo Ricci A. ${ }^{1}$, José Luis Troncoso J. ${ }^{1}$, Guillermo Avilés L. ${ }^{2}$, Raimundo Avilés D. ${ }^{3}$ \\ 1 Unidad de Ginecología, ${ }^{2}$ Unidad de Ultrasonografía Ginecológica, Departamento de Obstetricia y Ginecología, Clínica \\ Las Condes. ${ }^{3}$ Médico, Universidad Finis Terrae. Santiago, Chile.
}

\section{RESUMEN}

Presentamos un caso de embarazo cornual o intersticial diagnosticado en forma precoz a través de ecografía transvaginal, tratado exitosamente, en forma conservadora, con dosis única sistémica de metotrexato.

\section{PALABRAS CLAVE: Embarazo ectópico, embarazo cornual, embarazo intersticial, metotrexato}

\section{SUMMARY}

We present a case of cornual or interstitial pregnancy diagnosed during early stage through transvaginal ultrasound, treated successfully with single systemic intramuscular methotrexate dose.

\section{KEY WORDS: Ectopic pregnancy, cornual pregnancy, interstitial pregnancy, methotrexate}

\section{INTRODUCCIÓN}

El embarazo cornual o intersticial, es una variedad rara del embarazo ectópico y sus resultados pueden ser catastróficos (1-4). Su incidencia es de 1 por cada 2.500 a 5.000 recién nacidos vivos $(5,6)$. Correspondiendo a un 2 a $4 \%$ de los embarazos ectópicos en general (1). Se define como la implantación de un saco gestacional en el cuerno del útero, en la porción intersticial de la trompa de Falopio, adyacente al ligamento redondo (5). El embarazo cornual suele ser de más riesgo que las otras localizaciones ectópicas, ya que la ruptura del cuerno uterino puede provocar una hemorragia severa que en ocasiones puede ser causa de muerte materna $(3,6)$.

El mejor tratamiento para el embarazo ectópico cornual, aún no se ha definido, sin embargo, lo más importante es evitar su complicación (2). Para ello, lo más importante es realizar un diagnóstico precoz, para evitar la emergencia médica.

El objetivo de esta comunicación es presentar un caso de diagnóstico precoz de embarazo ectópico cornual, tratado eficazmente con dosis única de metotrexato por vía intramuscular.

\section{Caso clínico}

Mujer de 43 años, nulípara, con antecedentes de ooferectomía derecha por endometriosis, que acude a control por amenorrea de 8 semanas, con prueba positiva para $\mathrm{BHCG}$ en orina.

Se realizó ecografía transvaginal que demostró un útero en anteversoflexión, globalmente aumentado de tamaño (longitudinal $122 \mathrm{~mm}$, transversal 104 $\mathrm{mm}$, anteroposterior $94 \mathrm{~mm}$ ), con características de adenomiosis más acentuada en la cara anterior. En la región cornual izquierda, imagen compatible con saco gestacional de $39 \times 16 \times 26 \mathrm{~mm}$, con saco vitelino de $12 \mathrm{~mm}$ y embrión de $3,1 \mathrm{~mm}$ sin actividad cardíaca. Endometrio de $9 \mathrm{~mm}$. Ovario derecho ausente (antecedente ooferectomía). Ovario izquierdo de aspecto normal, con volumen de 6,6 cc (Figuras 1 - 3). 


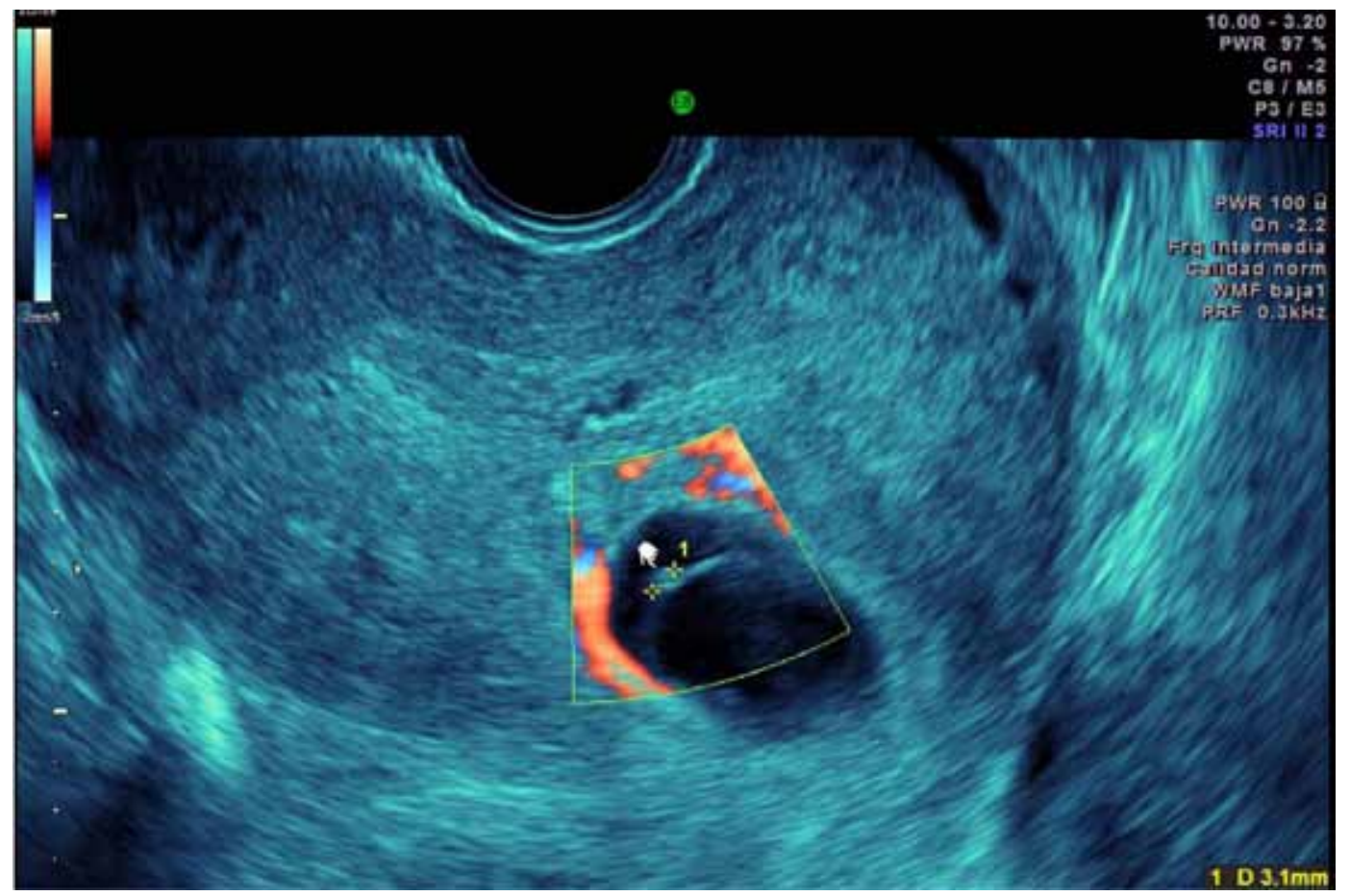

Figura 1. Visión de saco gestacional y embrión en cuerno uterino izquierdo. Se aprecia la cavidad endometrial vacía.

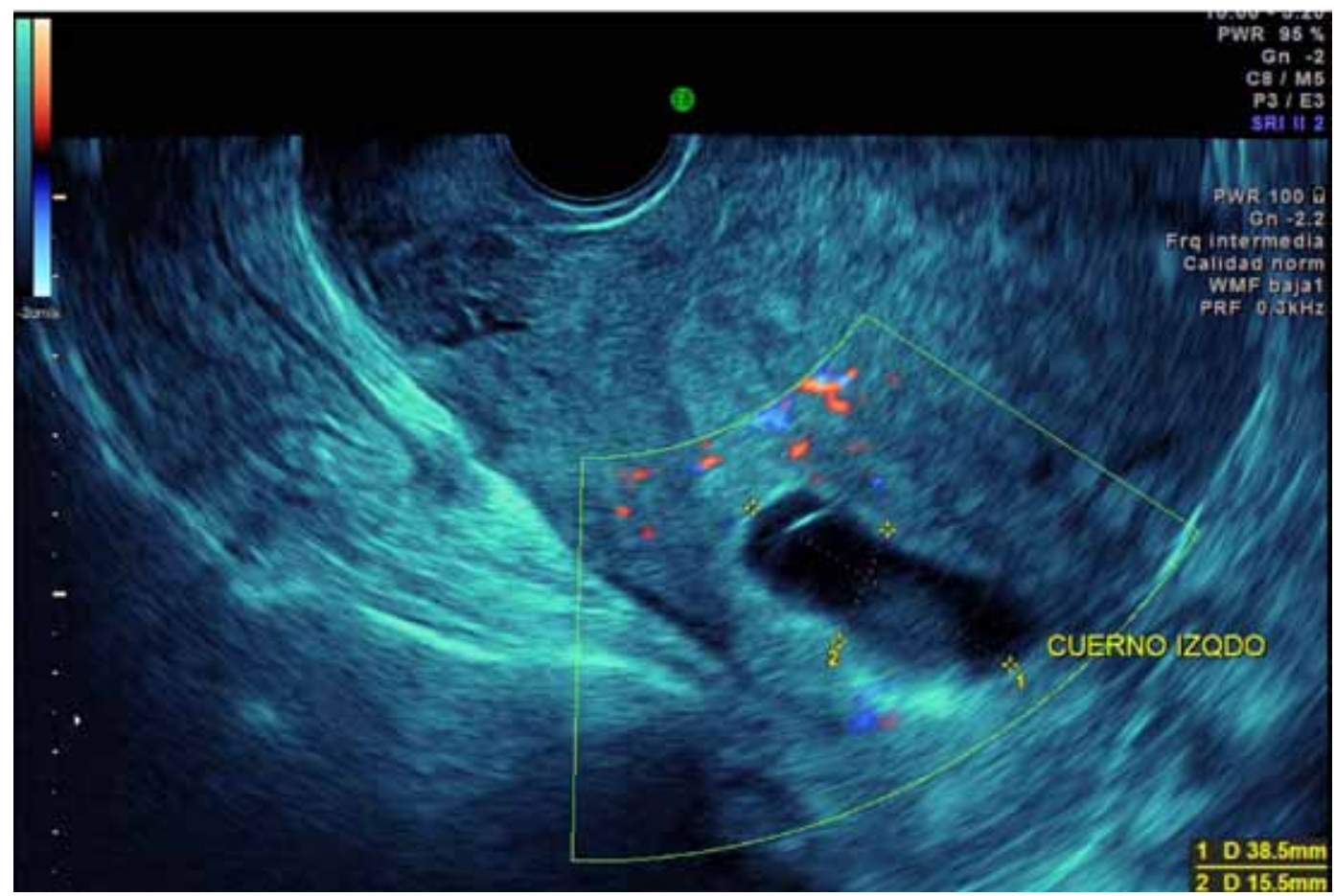

Figura 2. Visión de saco gestacional en cuerno uterino izquierdo. 


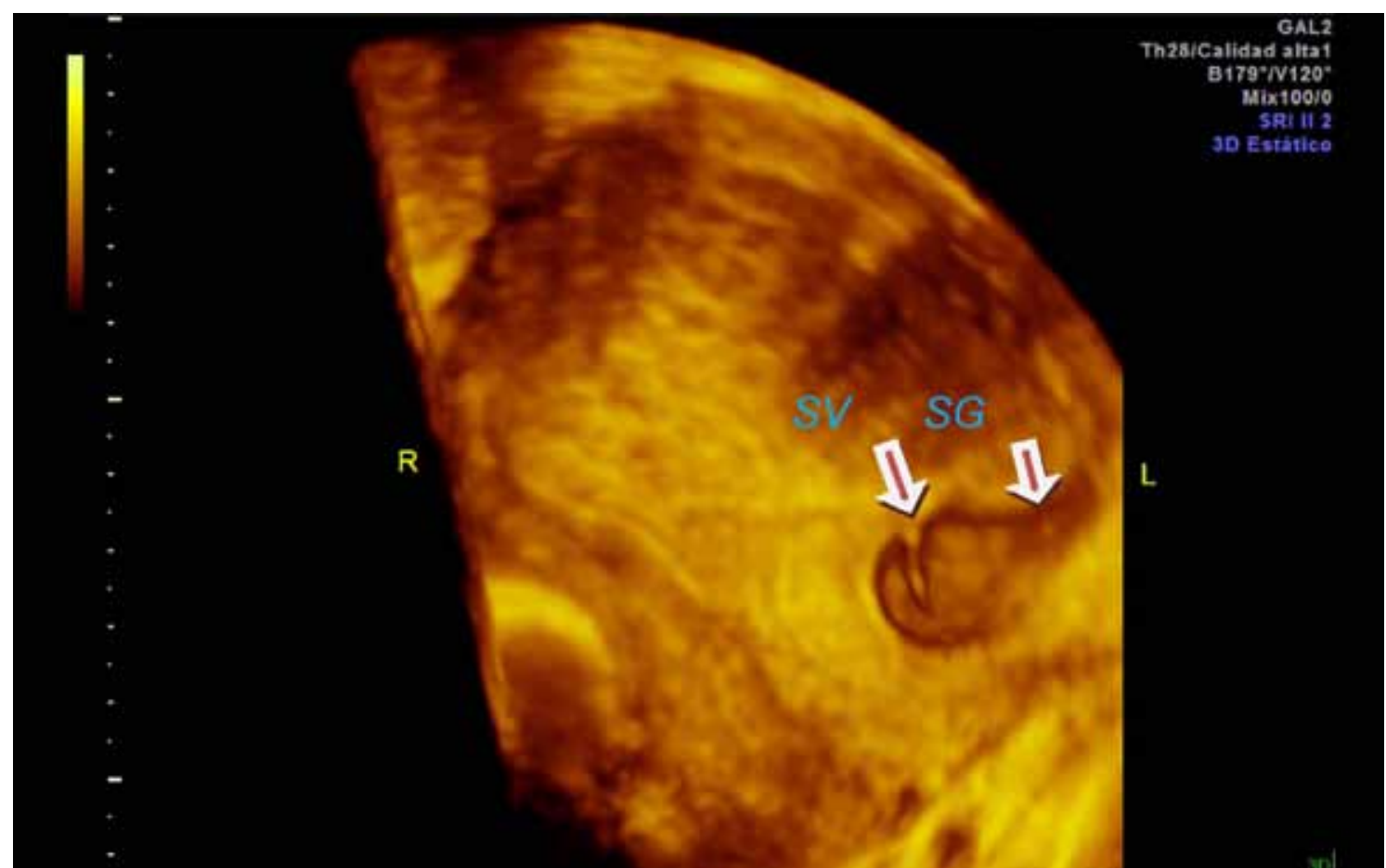

Figura 3. Visión con ultrasonido 3-D de saco saco vitelino (SV) y gestacional (SG) en cuerno uterino izquierdo

En base a los hallazgos anteriores, se realiza el diagnóstico de embarazo ectópico intersticial o cornual izquierdo, no complicado. Se explican a la paciente las alternativas terapéuticas y con el consentimiento de ella, se decide realizar una terapia conservadora, con metotrexato. Se aplica una inyección intramuscular de $100 \mathrm{mg}$, dosis calculada a $50 \mathrm{mg}$ por metro cuadrado de superficie corporal, según nomograma de Dubois (7). Previamente se controló la BHCG, resultando en 15.424 IU/I. Todos los exámenes sanguíneos de rutina se encontraban dentro de límites normales. Posterior a la medicación, a las 5 horas inicia sangrado vaginal con características similares a menstruación, abundante, por 5 días, para luego disminuir progresivamente hasta completar los 10 días. A los siete días del primer control, la BHCG fue de $7.206 \mathrm{IU} / \mathrm{l}$, a los 14 días de $1.523 \mathrm{IU} / \mathrm{l}$, a los 21 días de $159 \mathrm{IU} / \mathrm{l}$, y a los 28 días $0 \mathrm{IU} / \mathrm{l}$. Durante este proceso la paciente no presentó otros signos ni síntomas atribuibles a complicación del embarazo cornual.

\section{DISCUSIÓN}

La importancia de diagnosticar precozmente el embarazo ectópico intersticial o cornual, es principalmente debido a que es la única manera de evitar la más temida de las complicaciones, como es la hemorragia masiva debido a la ruptura uterina, que puede resultar en muerte materna, que se registra entre el 2 a $5 \%$ de los casos. Lo que resulta de 2 a 3 veces más frecuente que en el embarazo tubario $(1,5,8)$.

Para este diagnóstico, es indispensable la ecografía precoz, que debemos realizar en todos los embarazos, invariablemente, para detectar ubicaciones ectópicas como en este caso, registrar vitalidad embrionaria y hacer cálculos de edad gestacional. Las rupturas uterinas como complicación del embarazo cornual ocurren preferentemente en el segundo trimestre. Por esta razón es tan importante un diagnóstico precoz.

La porción del cuerno uterino o intersticial de la trompa de Falopio, es la parte de esta última que se encuentra dentro de la pared muscular del útero. Es de $0,7 \mathrm{~mm}$ de ancho y de $1 \mathrm{a} 2 \mathrm{~cm}$ de largo (8). Característicamente el embarazo ubicado en esta zona, se registra en la ecografía como un saco gestacional que interrumpe la llamada "línea intersticial" que corresponde a la unión del endometrio decidualizado con la mucosa tubárica. A esto se le ha llamado "signo de la línea intersticial" (1). Además, se ve un saco gestacional rodeado de miometrio delgado $(5,9,10)$. 
Los criterios diagnósticos son: cavidad uterina vacía, visión de un saco gestacional a más de 1 centímetro del borde más lateral de la cavidad uterina y presencia de una capa delgada de miometrio que rodea el saco. Tulandi y cols (8), demostraron en 2004, en una revisión de 32 embarazos cornuales, una sensibilidad del $80 \%$ y una especificidad del $99 \%$, en el diagnóstico ecográfico. Creemos que esto puede mejorar aún más con el apoyo del ultrasonido en 3-D (Figura 3).

El crecimiento de un saco gestacional en esta zona delgada y la penetración del tejido trofoblástico, provocará la ruptura del tejido miometrial, que en este territorio es abundantemente irrigado por la rama ascendente de la arteria uterina. Por esta razón, es altamente probable que la complicación de un embarazo en esta zona, con una ruptura uterina, provoque una hemorragia masiva. Una vez realizado el diagnóstico es poco aconsejable una actitud expectante o pasiva. De esta forma lo aconsejable es un tratamiento precoz y no uno de emergencia, cuando la paciente está sangrando, con inestabilidad hemodinámica secundaria y con alto riesgo vital.

En la actualidad no existe consenso en cuanto a cuál es el tratamiento más aconsejable. Podemos dividirlos en quirúrgicos, no quirúrgicos y la combinación de ellos. Entre los primeros se han descrito desde la resección del cuerno por laparotomía o laparoscopia (electrocoagulación, EndoloopMR, EndoGiaMR, etc.), cuernostomía con legrado cornual, incisión miometrial con aspiración del trofoblasto y la evacuación guiada por histeroscopia (11-15). Entre los tratamientos combinados, está la inyección intrasacular de metotrexato o de cloruro de sodio, guiado por histeroscopia y/o ultrasonido.

El tratamiento conservador es posible realizarlo solamente en casos con diagnóstico ecográfico precoz, embrión sin latidos, sin signos de complicación y estabilidad hemodinámica. Este tratamiento conservador, puede realizarse con uso de metotrexato. Sin embargo, debemos tener presente que tampoco está exento de riesgos o efectos secundarios. Se han descrito desde gastritis, mucositis, dermatitis, alopecía hasta inmunosupresión. Este riesgo se minimiza con el uso de monodosis, tal como ha sido demostrado para embarazos tubarios (16). Se utilizará repetición de dosis sólo cuando no hay descenso de la BHCG. Un estudio prospectivo, publicado por la Escuela de Medicina de Saint George, Londres, en 17 mujeres portadoras de embarazo cornual, tratadas con dosis única de metotrexato intramuscular, demostró un $94 \%$ de éxito, incluyendo cuatro casos con actividad cardíaca presente. Solamente 6 de ellas requirieron una segunda dosis. Estas últimas correspondieron a mujeres con BHCG sobre 5.000 IU/l al inicio (17). El "Royal College of Obstetrician and Gynaecologist" recomienda uso de metotrexato sistémico en pacientes con embarazo tubario, con niveles de
BHCG menor a 5.000 IU/l, sin signos de complicación y ausencia de latidos cardíacos embrionarios. Se repite una segunda dosis, a los siete días, si no hay descenso de la BHCG mayor al $15 \%$, entre el día 4 y 7 (18). En caso de falla del tratamiento médico, se ha descrito desde la intervención quirúrgica hasta el uso de embolización de la rama ascendente de la arteria uterina, provocando la necrosis del tejido trofoblástico. Con lo último, se trataría de preservar la función reproductiva $(2,19)$.

Respecto a fertilidad futura, cuando se ha realizado cirugía, debemos tener en cuenta la presencia de una cicatriz y la posible ruptura en un eventual embarazo posterior. Sin embargo, existe poca literatura médica respecto a casos con tratamiento conservador. Algunos sostienen que después de un tratamiento médico, incluso con comprobación de normalidad anatómica vía histerosalpingografía y visión directa por histeroscopia, el riesgo de un nuevo embarazo cornual permanece alto $(1,20)$. Esto sería debido a que otras causas que contribuyeron a la misma implantación cornual en la primera ocasión, probablemente seguirían sin variación.

\section{CONCLUSIÓN}

El embarazo cornual o intersticial es una localización ectópica poco frecuente, con alta morbimortalidad materna por hemorragia masiva. Por lo anterior, debemos estar atentos a realizar un diagnóstico precoz para evitar sus complicaciones. Al realizar un diagnóstico temprano, el uso de metotrexato representa una opción de tratamiento médico conservador, eficaz, evitando una intervención quirúrgica compleja y de alto costo.

\section{REFERENCIAS}

1. Faraj R, Steel M. Review Management of cornual (interstitial) pregnancy. The Obstetrician \& Gynaecologist. 2007;9:249-55.

2. Szylit NA, Podgaec S, Traina E, Oliveira Rde C. Video laparoscopic intervention for an interstitial pregnancy after failure of clinical treatment. Sao Paulo Med J. 2012;130:202-7.

3. Takei T, Matsuoka S, Ashitani N, Makihara N, Morizane M, Ohara N. Ruptured cornual pregnancy: case report. Clin Exp Obstet Gynecol 2009;36:130-2.

4. Gallegos F, Pavéz C, Jara M, Jesam C, Montero JC, Bustos JC. Embarazo ectópico intersticial complicado: urgencia ginecológica. Rev Obstet Ginecol 2005;70:414-17.

5. Luengo-Tabernero A, Zornoza-García V, Luengo-Harto S, Lajas-Susaño JA. Embarazo ectópico cornual. Tratamiento eficaz con dosis única de metotrexato. Clin Invest Gin Obst 2009;36:190-2.

6. Carazo Hernández B, Pérez-Ezquerra BR, Sanz López A, Garcés Valenzuela M. Uterine rupture of a 
cornual pregnancy: an obstetric emergency. Ginecol Obstet Me. 2012;80:491-4.

7. Du Bois D, Du Bois EF. A formula to estimate the approximate surface area if height and weight be known. 1916. Nutrition. 1989;5(5):303-11; discussion 312-3.

8. Tulandi T, Al-Jaroudi D. Interstitial pregnancy: results generated from The Society of Reproductive Surgeons Registry. Obstet Gynecol 2004;103:47-50.

9. Ackerman TE, Levi CS, Dashefsky SM, Holt SC, Lindsay DJ. Interstitial line: sonographic finding in interstitial (cornual) ectopic pregnancy. Radiology 1993;189:83-7.

10. Advincula AP. Senapati S. Interstitial pregnancy. Fertil Steril 2004;82:1660-2.

11. Moawad NS, Dayaratna S, Mahajan ST. Mini-cornual excision: a simple stepwise laparoscopic technique for the treatment of cornual pregnancy. JSLS 2009;13:8791.

12. Moon HS, Choi YJ, Park YH, Kim SG. New simple endoscopic operations for interstitial pregnancies. Am J Obstet Gynecol 2000;182(1 Pt 1):114-21.

13. Sergent F, Le Cornec JB, Meilhaud MF, Marpeau L. Laparoscopic cornual excision with an automatic stapler for ruptured interstitial pregnancies. J Gynecol Obstet Biol Reprod (Paris) 2003;32:426-30.
14. Chan LY, Yuen PM. Successful treatment of ruptured interstitial pregnancy with laparoscopic surgery. A report of 2 cases. J Reprod Med 2003;48:569-71.

15. Minelli L, Landi S, Trivella G, Fiaccavento A, Barbieri F. Cornual pregnancy successfully treated by suction curettage and operative hysteroscopy. BJOG 2003;110:1132-4.

16. Stovall TG, Ling FW. Single dose methotrexate: an expanded clinical trial. Am J Obstet Gynecol 1993;168(6 Pt 1):1759-62.

17. Jermy K, Thomas J, Doo A, Bourne T. The conservative management of interstitial pregnancy. BJOG 2004;111:1283-8.

18. Kelly AJ, Sowter MC, Trinder J. The management of tubal pregnancy. Royal College of Obstetricians and Gynaecologists 2004; Guideline № 21;1-10.

19. Deruelle $P$, Lucot JP, Lions C, Robert Y. Management of interstitial pregnancy using selective uterine artery embolization. Obstet Gynecol 2006;107(2 Pt 1):427-8.

20. Van der Weiden RM, Karsdorp VH. Recurrent cornual pregnancy after heterotopic cornual pregnancy successfully treated with systemic methotrexate. Arch Gynecol Obstet 2005;273:180-1. 